\title{
Pharmacoepidemiological Profiles of Oral Hypoglycemic Agents in Elbanjadeed Hospital, Khartoum, Sudan
}

\section{Babiker Abadi AE ${ }^{1}$, Mousnad MA ${ }^{1 *}$, Altayeb $\mathrm{A}^{2}$ and Mahmoud $\mathbf{M}^{2}$}

${ }^{1}$ Department of Pharmacy Practice, Faculty of Pharmacy, International University of Africa (IUA), Sudan

${ }^{2}$ Fast International Medical Company, Sudan

*Corresponding author: Mohamed Awad Mousnad, Department of Pharmacy Practice, Consultant of Pharmacoeconomics and Pharmacoepidemiology, Assistant Professor, Faculty of Pharmacy, International University of Africa (IUA), Khartoum, Sudan, Email: m_abdalaziz@yahoo.com

\section{Research Article \\ Volume 3 Issue 2}

Received Date: April 13, 2020

Published Date: April 30, 2020

DOI: $10.23880 /$ jqhe-16000158

\section{Abstract}

Background: Diabetes is a metabolic disorder resulting in chronic microvascular and macrovascular complications. Diabetes affects an increasing number of patients, globally, by the year 2030, the number expected to more than double. Hypertension affects about $60 \%$ of patients with type 2 diabetes.

Aim: The present study was planned with the objective of the utilization of oral antidiabetic drugs in Elbanjadeed hospital in Khartoum North. The study also aimed to determine what is the consumption rate of oral antidiabetic agents in Elbanjadeed also to identify what are the most prescribing oral antidiabetic drugs in the hospital.

Methods: The study design was a cross-sectional prospective study design, drug utilization data was collected in 2013. ATC/ DDD methodology, WHO prescribing indicators were selected and used, collected data were analyzed using appropriate statistical techniques. As per the study criteria, data were collected from 150 prescriptions of diabetic patients who were admitted during the April and May 2013.

Main results: The study results showed that was predominant in male more than female. age group (41-60) was the active diabetic age group. Metformin was the most prescribed antidiabetic drug. Alimentary tract and Metabolism was the most prescribed drug class because it includes the antidiabetic drugs. The most disorders associated with diabetes was Cardiovascular disorder which affects about (27\%) of diabetic patients. Average cost was 115.8 (SDG) which was very high because the average number of drug per prescription was high. The average number of drugs per prescription was 4.3 and the percentage of generic drug prescribed was $66.7 \%$. The percentage of the number of drugs in EML was $60.1 \%$.

Conclusions: The study showed that male was predominant more than female, the active age group of diabetes was (41-60), metformin was the most prescribed oral antidiabetic and cardiovascular problems were the most disorder associated with diabetes.

Keywords: Diabetes Mellitus; Pharmacologic Therapy; Insulin

\section{Introduction}

\section{Background}

Diabetes Mellitus (DM) is a group of metabolic disorders (disease of the endocrine pancreas) characterized by hyperglycemia and abnormalities in carbohydrate, fat and protein metabolism. It results from defects in insulin secretion, insulin sensitivity or both. Chronic microvascular, macrovascular and neuropathic complications may occur [1]. They are the long-term complications of diabetes that are the main causes of morbidity and mortality. Diabetes is 
in danger of becoming almost pandemic [2].

Diabetes affects an increasing number of patients, globally, by the year 2030, the number expected to more than double. Hypertension affects about $60 \%$ of patients with type 2 diabetes. The direct health care costs of diabetes worldwide, for people in the 20-79 ages, are estimated to be at least 153 billion international dollars. The total direct health care expenditure worldwide on diabetes will be between 213-396 billion international dollars in 2025 [3].

\section{The three main types of diabetes are:}

1: Type I diabetes (Insulin-Dependent Diabetes); the cause appears to be a progressive autoimmune destruction of the pancreatic $\beta$ cells. Most instances of autoimmune pancreatic destruction are idiopathic but, some may occur following viral infections. Little or no insulin secretion occurs.

2: Type II Diabetes Mellitus (Non-Insulin-Dependent Diabetes); Greater prevalence than type I diabetes and it is due to insulin resistance which may be related to increased levels of free fatty acids (FFA) that stimulate insulin secretions and inhibit glucose uptake by Tissues. Type $1 \mathrm{DM}$ accounts for $5 \%$ to $10 \%$ of all diabetes cases; it generally develops in childhood or early adulthood and results from immunemediated destruction of pancreatic $\beta$-cells, resulting in an absolute deficiency of insulin. There is a long preclinical period (up to 9 to 13 years) marked by the presence of immune markers when $\beta$-cell destruction is thought to occur.

3: Type: Gestational diabetes mellitus (GDM) is defined as any degree of glucose intolerance with onset or first recognition during pregnancy.

Medical nutrition therapy is recommended for all patients. For type 1 the focus is on regulating insulin administration with a balanced diet to achieve and maintain a healthy body weight and meal plan that is moderate in carbohydrates and low in saturated fat. In patients with type 2 DM-Weight loss has been reported to improve insulin sensitivity Insulin. Aerobic exercise can improve insulin resistance and glycemic control in most patients and may reduce cardiovascular risk factors, contribute to weight loss or maintenance, and improve well-being. Dietary management and meal plan are also important for type $2 \mathrm{DM}$.

\section{Pharmacologic Therapy includes:}

1: Insulin and Other Injectable Preparations Including different preparations of insulin Rapid-acting insulin's, Shortacting insulin's, Intermediate-acting insulin's (NPH), Longacting insulin's, Premixed insulin's, and Other injectable preparations (Exenatide and Pramlintide).

\section{2: Oral Hypoglycemic Agents: Including different}

classifications: Sulfonylurea (e.g.: Chlorpropamide, Glibencalamide, Glimepiride and Gliclazide), Biguanides (e.g: metformin), Thiazolidinediones (e.g.: Pioglitazone), $\alpha$-Glucosidase inhibitors (e.g.: Acarbose), and Dipeptidyl peptidase-IV (DPP-IV) inhibitors (e.g: Sitagliptin). Also different combinations are available (e.g.: Rosiglitazone/ metformin and Glipizide/metformin) [1].

According to the Mediscor Medicines, et al. Review [3], diabetes mellitus type II was one of the top five most prevalent Chronic Disease List (CDL) conditions and accounted for $11.2 \%$ and $9.9 \%$ of the percentage of total CDL. Diabetes mellitus type I was ranked seventh and accounted for $4.9 \%$ and $1.6 \%$ of the total CDL. For Africa, the number of diabetes sufferers in Africa remains uncertain [4]. The diabetes population will double over the next twenty-five years in Africa according to WHO (World Health Organization) and the IDF (International Diabetes Federation) estimations [4]. The African continent accounts for 14 million people with diabetes, with Sub-Saharan African the region of 7 million people with about 2.5 million of them in the Southern African regions [3].

Diabetes in Sudan is one of the highest mortality rates for a non-infectious disease and it is the most common cause of hospital admission and morbidity [5]. The problems in Sudan are that the actual number of people with diabetes in Sudan is unknown and large scale studies in Sudan are absence, also Health services in the form of care centres are only available in two small centres in Khartoum [5]. According to WHO diabetes in Sudan represent 2\% of the total mortality death in all ages, also they are said that the mortality estimates for Sudan have a high degree of uncertainty because they are not based on any national NCD mortality data and there are no records for many metabolic risk factors such as: raised blood glucose, obesity and raised cholesterol [6].

Diabetes affects an increasing number of patients, and the number is expected to reach more than double, worldwide, by the year 2030 [7].

The World Health Organization (WHO) Eastern Mediterranean Region identified to be the highest prevalence of diabetes globally. More than 15\% diabetes prevalence in 7 countries in this Re-gion (Kuwait, Lebanon, Qatar, Saudi Arabia, Bahrain, United Arab Emirates and Egypt), a medium prevalence of $9-12 \%$ in other 7 countries (Iraq, Jordan, Oman, Sudan, Syrian Arab Re-public, Tunisia and Yemen) while below 9\% prevalence only in 6 countries (Algeria, Comoros, Djibouti, Mau-ritania, Morocco and Somalia).

Presentation of the utilization of these drugs is important because it evaluates factors related to the prescribing, dispensing, administering, taking of medication, 
and its associated events (either beneficial or adverse) of oral hypoglycemic agents. The impact of diabetes on health care expenditures has been increasingly proved, several 'cost of illness' studies have shown a three-fold increase in the direct costs of diabetic patients compared with non-diabetic patients in the setting of different health care systems. Type 2 diabetes, therefore, has increasingly become the main cause of growing costs in hospitalization and drugs because of complication. The excess costs were largely due to an increased number of hospital days, expensive outpatient's treatments, higher costs for nursing home care and increased drug consumption. Many problems facing Diabetes care in Sudan. These include lack of efficient diabetes care centre, lack of specially trained personnel, high cost of antidiabetic drugs, poor compliance of diet or therapy and lack of accurate reporting system. Little is known about medicine expenditures and utilization in Elbanjadeed hospital in Sudan capital, Khartoum. This study was conducted to heighted antidabetic utilization trends and to provide information for policy design and recommend areas for interventions. This research provides a baseline data for future studies concerning medicine in Elbanjadeed hospital. The findings will help in the development and application of statistics on medicines for drug prescription and provide better consumption presentation, improve the quality of statistics on medicines, facilitate national and international comparisons of medicine utilization.

This research generally aims to describe oral antidiabetic utilization trends in Elbanjadeed hospital and specifically to

- Describe and compare the distribution of prescribed drugs to diabetic patients.

- Determine the prescribing prevalence of antidiabetic and related co- morbidities drugs

- Determine the patterns of oral antidiabetic drug consumptions.

- Estimate the average costs per prescription of DM in Elbanjadeed hospital.

\section{Methodology}

\section{Study Design}

Quantitative cross-sectional prospective study design. Prescription data from outpatient and inpatient pharmacy in Elbanjadeed hospital in Khartoum North, Sudan were collected and used in this study. A total of 150 prescriptions were collected via structured data collection forms in the period from April to May, 2013.

\section{Inclusion Criteria}

Only prescriptions with oral antidiabetic drug were included. The data were collected from outpatients and inpatients pharmacy. The research was focused on over 18year diabetics' patients.

\section{Exclusion Criteria}

Patients taking insulin were excluded, children were excluded and patient's complications were excluded.

\section{Study Instruments and Tools}

ATC Classification: The oral antidiabetic list was classified according to the Anatomical Therapeutic Chemical (ATC) classification system [8].

ATC/DDD Methodology: The utilization expressed as the number of defined daily doses per 1000 inhabitants per day (DDD/1000/day) or DID as recommended by the WHO Working Group on Drug Utilization [9].

Referee to Appendix B for DDD calculations.

WHO Drug Use Indicators: Selected drug use indicators as suggested by WHO/INRUD used in this study [9].

A: Core drug use indicators: Prescribing indicators

- Average number of drugs per encounter.

- Percentage of drugs prescribed by generic name.

- Percentage of drugs prescribed from essential list or formulary.

B: Complementary drug use indicators: 5-Average drug cost per encounter.

Referee to Appendix B for WHO Drug Use Indicators calculations.

\section{Data Collection Procedure}

Prescriptions data was collected from the using valid data collection forms. These forms contain details in Drug information consisting of names of the drug prescribed, strength, dosage, duration, prescribers and registration. 150 prescriptions were collected in the time between $1 / 4 / 2013$ 31/5/2013.

\section{Data Analysis}

The data entering process and all dependent variables calculations required utilized the Microsoft Excel 2007 software. Then after the data was organized, it transferred for analysis using the SPSS software (Statistical Package for Social Sciences version). 
Descriptive analysis, cross-tabulation and frequency distribution were used to describe and compare the patient's characteristics, prescription practices and drug consumption. An inferential statistical test (Chi-square test; alpha value $=0.05$ ) was used for testing the significance of the observed differences between variables. Pearson's correlation coefficient was used to indicate any association between the increase in medical expenditure and independent factors (population coverage and the number of patients). Analysis of variance (ANOVA), one-way was used to measure mean differences of the numerical variables between groups.

\section{Ethical Approval}

Ethical approval was obtained from the Ethics Committee (IEC) of Elban jadeed hospital in Khartoum North, Sudan.

\section{Results}

Description of the Study Population

\begin{tabular}{|l|c|c|}
\hline & Characteristics & Frequency (\%) \\
\hline \multirow{2}{*}{ Gender } & Male & $325(52.1)$ \\
\cline { 2 - 3 } & Female & $299(47.9)$ \\
\hline \multirow{3}{*}{ Age group } & $20-40$ & $19(3.00)$ \\
\cline { 2 - 3 } & $41-60$ & $408(65.40)$ \\
\cline { 2 - 3 } & More than 60 & $197(31.60)$ \\
\hline
\end{tabular}

Table 1: Summary of patient's characteristic.

The age group (41-60) was age group of the highest frequency (65.4\%) followed by age group more than 60
(31.6\%) and lowest age frequency was for (20-40) which was (3\%).

\section{Age-Sex Specific of Diabetes Prevalence}

\begin{tabular}{|c|c|c|c|}
\hline Age group & Female & Male & Total \\
\hline age $20-40$ & $3(1.3 \%)$ & $7(3.1 \%)$ & $10(4.5 \%)$ \\
\hline age $41-60$ & $80(35.7 \%)$ & $61(27.2 \%)$ & $141(62.9 \%)$ \\
\hline age more than 60 & $29(12.9 \%)$ & $44(19.6 \%)$ & $73(32.6 \%)$ \\
\hline Total & $\mathbf{1 1 2}(50.0 \%)$ & $112(50.0 \%)$ & $\mathbf{2 2 4}(100.0 \%)$ \\
\hline
\end{tabular}

Table 2: The age group distribution of diabetes prevalence in male and female patients in April 2013.

\begin{tabular}{|c|c|c|c|c|}
\hline Age group & Female & Male & Total & *p value \\
\hline age $20-40$ & $4(1.0 \%)$ & $5(1.2 \%)$ & $9(2.2 \%)$ & \multirow{2}{*}{0.001} \\
\hline age $41-60$ & $142(35.5 \%)$ & $125(31.2 \%)$ & $267(66.8 \%)$ & \\
\cline { 1 - 4 } age more than 60 & $41(10.2 \%)$ & $83(20.8 \%)$ & $124(31.0 \%)$ & \\
\hline Total & $\mathbf{1 8 7}(46.8 \%)$ & $\mathbf{2 1 3}(53.2 \%)$ & $\mathbf{4 0 0}(100.0 \%)$ & \\
\hline
\end{tabular}

Table 3: The age group distribution of diabetes prevalence in male and female patients in May, 2013.

In age group (20-40) the distribution of male was more than female in both April ( male (3.1\%), female (1.3\%)) and May (male $(1.2 \%)$, female $(1.0 \%)$ ), but in age group (41-60) female was more distributed than male in the April (female (35.7\%), male (27.2\%) ) and May (female (35.5\%), male(31.2\%) ). In age group more than 60 male was more distributed than female in April (male (19.6\%), female (12.9\%) and May (male (20.8\%) female (10.2\%). 


\begin{tabular}{|c|c|c|}
\hline \multirow{2}{*}{ Drug } & \multicolumn{2}{|c|}{ Total utilization } \\
\cline { 2 - 3 } & April & $31(64.6 \%)$ \\
\hline Metformin & $17(35.4 \%)$ & $11(50.0 \%)$ \\
\hline Glibencalamide & $11(50.0 \%)$ & $12(66.7 \%)$ \\
\hline Glimperide & $6(33.3 \%)$ & $18(85.7 \%)$ \\
\hline Gliclazide & $3(14.3 \%)$ & $4(100 \%)$ \\
\hline Pioglitazone & $0(.0 \%)$ & $\mathbf{1 6 4 ( 6 5 . 3 \% )}$ \\
\hline Total & $\mathbf{8 7}(\mathbf{3 4 . 7 \% )}$ & \\
\hline
\end{tabular}

Table 4: The prescribing frequency of the oral antidiabetic drugs in April and May, 2013.

Utilization of Metformin (64.6\%) , Glimperide (66.7\%) , Gliclazide (85.7\%) , and Pioglitazone (100\%) was more in May more than April Metformin (35.4\%), Glimperide
(33.3\%), and Pioglitazone (.0\%). Glibencalamide utilization was similar in both April and May which was (50.0\%).

\begin{tabular}{|c|c|c|c|c|}
\hline Drug name & Male & Female & Total & p value \\
\hline Metformin & $1(21.8 \%)$ & $2(28.7 \%)$ & $44(50.6 \%)$ & \\
\cline { 1 - 4 } Glibencalamide & $12(5.7 \%)$ & $11(12.6 \%)$ & $23(26.4 \%)$ & 0.429 \\
\hline Glimperide & $5(5.7 \%)$ & $12(13.8 \%)$ & $17(19.5 \%)$ & $3(3.4 \%)$ \\
\hline Gliclazide & $1(1.1 \%)$ & $2(2.3 \%)$ & $\mathbf{8 7}(\mathbf{1 0 0 . 0 \% )}$ & \\
\hline Total & $\mathbf{3 7 ( 4 2 . 5 \% )}$ & $\mathbf{5 0}(\mathbf{5 7 . 5 \% )}$ & \\
\hline
\end{tabular}

Table 5: The prescribing differences of oral hypoglycemic agents between male and female patients in April, 2013.

\begin{tabular}{|c|c|c|c|c|}
\hline Drug name & Male & Female & Total & p value \\
\hline Metformin & $44(26.8 \%)$ & $38(23.2 \%)$ & $82(50.0 \%)$ & \\
\cline { 1 - 4 } Glibencalamide & $14(8.5 \%)$ & $12(7.3 \%)$ & $26(15.9 \%)$ & \multirow{2}{*}{0.802} \\
\hline Glimperide & $11(6.7 \%)$ & $16(9.8 \%)$ & $27(16.5 \%)$ & $18(11.0 \%)$ \\
\hline Gliclazide & $12(3.7 \%)$ & $6(3.7 \%)$ & $11(6.7 \%)$ & \\
\hline Pioglitazone & $6(3.7 \%)$ & $5(3.0 \%)$ & $\mathbf{1 6 4}(\mathbf{1 0 0 . 0} \%)$ & \\
\hline Total & $\mathbf{8 7}(\mathbf{5 3 . 0 \% )}$ & $\mathbf{7 7 ( 4 7 . 0 \% )}$ & \\
\hline
\end{tabular}

Table 6: The prescribing differences of oral hypoglycemic agents between male and female patients in May, 2013.

In April metformin was prescribed in female more than male but, in May it was prescribed in male more than female. Glibenclamide in April and May was prescribed in male more than female. Glimperide in April and May was prescribed in female more than male. In April Gliclazide was prescribed in female more than male, but in May it was prescribed in male more than female. There was no prescribing of Pioglitazone in April but in May it was prescribed in male more than female.

\section{Prescribing Frequency of Different Drug Classes among Age Groups}

The highest prescribing frequency was in Alimentary tract and metabolism class (53.4\%) followed by cardiovascular system class and then Blood and blood forming organs class $(17.5 \%)$. The lowest prescribing frequency was in Nervous system $(0.2 \%)$ and Respiratory system $(0.2 \%)$ drug classes. 


\begin{tabular}{|c|c|c|c|c|c|}
\hline Drug class & $\mathbf{2 0 - 4 0}$ & $\mathbf{4 1 - 6 0}$ & More than $\mathbf{6 0}$ & Total & p value \\
\hline Alimentary tract and metabolism & $8(3.6 \%)$ & $85(37.9 \%)$ & $30(13.4 \%)$ & $123(54.9 \%)$ & $28(12.5 \%)$ \\
\hline Blood \& Blood forming Organs & $1(.4 \%)$ & $19(8.5 \%)$ & $8(3.6 \%)$ & $65(29.0 \%)$ \\
\hline Cardiovascular system & $1(.4 \%)$ & $36(16.1 \%)$ & $28(12.5 \%)$ & $1(.4 \%)$ \\
\hline Genito urinary system and sex hormones & - & - & $1(.4 \%)$ & $2(.9 \%)$ \\
\hline $\begin{array}{c}\text { Systemic hormonal preparations, excl. sex } \\
\text { hormones and insulins }\end{array}$ & - & - & $2(.9 \%)$ & 0.094 \\
\hline Musculo-skeletal system & - & - & $1(.4 \%)$ & $1(.4 \%)$ \\
\hline Nervous system & - & - & $1(.4 \%)$ & $1(.4 \%)$ \\
\hline Respiratory system & - & $1(.4 \%)$ & - & $1(.4 \%)$ \\
\hline Sensory organs & - & - & $2(.9 \%)$ & $2(.9 \%)$ \\
\hline Total & $10(4.5 \%)$ & $141(62.9 \%)$ & $73(32.6 \%)$ & $224(100.0 \%)$ \\
\hline
\end{tabular}

Table 7: Prescribing frequency of different drug classes among age groups in April, 2013.

\begin{tabular}{|c|c|c|c|c|c|}
\hline Drug class & $20-40$ & 41-60 & More than 60 & Total & p value \\
\hline Alimentary tract and metabolism & $8(2.0 \%)$ & 148(37.) & $54(13.5 \%)$ & $210(52.5 \%)$ & \multirow{10}{*}{0.047} \\
\hline Blood \& Blood forming Organs & $1(.2 \%)$ & $57(14.2 \%)$ & $23(5.8 \%)$ & $81(20.2 \%)$ & \\
\hline Cardiovascular system & - & $61(15.2 \%)$ & $43(10.8 \%)$ & $104(26.0 \%)$ & \\
\hline Genito urinary system and sex hormone & - & - & $1(.2 \%)$ & $1(.2 \%)$ & \\
\hline $\begin{array}{l}\text { Systemic hormonal preparations, excl. sex } \\
\text { hormones and insulins }\end{array}$ & - & - & - & $0(0.0 \%)$ & \\
\hline Musculo-skeletal system & - & $1(.2 \%)$ & $2(.5 \%)$ & $3(.8 \%)$ & \\
\hline \multirow{2}{*}{ Nervous system } & \multirow{2}{*}{-} & \multirow{2}{*}{-} & \multirow{2}{*}{ - } & 0 & \\
\hline & & & & $0.00 \%$ & \\
\hline Respiratory system & - & - & - & $0(0.0 \%)$ & \\
\hline Sensory organs & - & - & $1(.2 \%)$ & $1(.2 \%)$ & \\
\hline
\end{tabular}

Table 8: Prescribing frequency of different drug classes among age groups in May, 2013.

For the Alimentary tract and metabolism class the distribution in the two months were high in the age group $(20-40)$ which were $(37.9 \%)$ and $(37.0 \%)$ respectively. In Blood \& Blood forming Organs class the distribution was increased from (12.5\%) in April to (20.2\%) in May. Similar percentage for the Genito urinary system and sex hormones class for April and May which was (0.2\%) and there was no prescribing for Systemic hormonal preparations, excl. sex hormones and insulin in May. Prescribing frequency for the Nervous system was decreased from $(0.4 \%)$ in April to $(0.0 \%)$ in May, these also occur for Respiratory system frequency from (0.4\%) in April to (0.0\%) in May, and Sensory organs frequency from $(0.9 \%)$ in April to $(0.2 \%)$ in May. Prescribing for the Respiratory system class was in April and no prescribing in May.

\section{Summary and Profile of Antidiabetics Utilization}

\begin{tabular}{|c|c|c|c|c|c|c|}
\hline \multirow{2}{*}{ Drug name } & \multirow{2}{*}{ DDD (mg) } & \multirow{2}{*}{ ATC Classification } & \multicolumn{2}{|c|}{ DDDs } & \multicolumn{2}{c|}{ DID } \\
\cline { 4 - 7 } & & & April & May & April & May \\
\hline Metformin & 2000 & (A10BA02) & 470.2 & 1181.6 & 0.02 & 0.06 \\
\hline Glibenclamide & 10 & (A01BB12) & 367.3 & 1676.6 & 0.02 & 0.08 \\
\hline Glimperide & 60 & (A01BB12) & 1560 & 5880 & 0.08 & 0.29 \\
\hline Gliclazide & 60 & (A10BB09) & 2773.3 & 4950.9 & 0.14 & 0.25 \\
\hline Pioglitazone & 30 & (A10BG03) & 0 & 9800 &. & 0.49 \\
\hline
\end{tabular}

Table 9: Defined daily dose and Defined daily dose per 1000 inhabitants in April and May. 


\section{Antidiadetic Utilization Trends}

In general, the consumption of antidiabetic in the Elbanjadeed Hospital was increased from 0.07 DID in April to 0.23 DID in May. In other words, the utilization of antidiabetics was greater in May than April (0.07 DID vs. 0.23 DID). The consumption by individual antidiabetics of Metformin was increased from 470.2 DDDs in April to 1181.6 DDDs in May, while for Glibenclamide utilization increased from 367.3 DDDs in April to 1676.6 DDDs in May. As for Glimperide utilization increased from 1560 DDDs in April to 5880 DDDs in May while for Gliclazide utilization increased from 2773.3DDDs in April to 4950.9 DDDs in May. There was no utilization of Pioglitazone in April while it was present in May as 9800 DDDs.

\section{WHO Selected Drug Use Indicators}

\begin{tabular}{|c|c|}
\hline Indicator & Result \\
\hline Average number of drug per prescription & 4.3 \\
\hline Percentage of generic drug prescribed & $66.70 \%$ \\
\hline Percentage no. of drugs in EML & $60.10 \%$ \\
\hline Average drug cost per prescription (RM) & 115.8 \\
\hline
\end{tabular}

Table 10: Selected drug use indicators results for the study.

\section{Differences in Medicine Utilization Between April And May, 2013}

To examine the differences between medicine utilization among the two months, a parametric statistical test oneway ANOVA, was used. There was statistically significant difference between groups as determined by one-way ANOVA ( $\mathrm{P}=0.000)$.

\section{Correlation between Medicine Utilization and Population Characteristics}

To examine the exact relationships between the medicine expenditures and population characteristics (age-gender), the Pearson correlation coefficient test was used. The results showed a very weak positive correlation $(r=0.14, p<0.001)$ with the age; and weak positive correlation $(r=0.14, p<0.001)$ with gender.

\section{Discussion}

In this study the age groups (41-60) years old was of the highest frequency while the least frequency age group was (20-40) years old with a percentage. These findings indicate that DM was more towards the active age group (20-40) years old. Different findings were reported in a study conducted by Upadhyay DK, et al. [10] in Nepal where the greatest number found were in the age group of 51-60 years followed by 61-70 years and the least frequency was for 21-30 years. Different findings also reported in study by Dianne L, et al. [12] in U.S were between 40 and 59 years old while over half frequency for the age $>65$ yr. Similar findings were reported in study by Mousnad, et al. [13] in Malaysia.

In this study male was higher than female. In a study conducted by Leelavathi D, et al. [14] in Manipal majority of the patients were males, that was in 2008, 2009 and 2010 respectively. A study conducted by Upadhyay DK, et al, [10] in Nepal males were more than females. There was a study conducted in Nigeria by En were, et al. [15] showed that Females with diabetes mellitus were more males. These differences between results may be due to geographical difference and selection of patients from single center compared to other countries where they used data base for the study.

Prescribing frequency of Alimentary tract and metabolism drug class was which the highest frequency was because it is including antidiabetics which were the focus of our study. Cardiovascular system drug class frequency was high indicating that cardiovascular diseases were the most complications associated with diabetes. In another study conducted by Leelavathi D, et al. [14] in Manipal cardiovascular diseases was more than half. Less percentage for respiratory and nervous system disorders associated with diabetes in this study. Prescribing frequency of Alimentary tract and metabolism class and cardiovascular system drug class were the highest in age group (41-60) in both April and May. There were little problems of respiratory and nervous system problem associated with diabetes this was confirmed by the smallest percent of both classes in April and May.

Although distribution of both gender within age groups in this study was highest in age (41-60) in April and May, female was distributed more than male in both months. But a study conducted by Lisha, et al. [16] in Ajman male was distributed more than female in age (45-60).

The most prescribed oral antidiabetic drug was metformin in both April and May. Absence of pioglitazone in April and presentin May was due to introduced of pioglitazone as new intervention in Elbanjadeed Hospital. There was a high difference in prescription of oral antidiabetics between April and May which was high in May more than April there may be due to poor adherence of using standard prescribing guidelines by prescribers or there was a frequent change in prescribers with different knowledge.

Metformin was prescribed for more than half of diabetic patient in April and for half of diabetic patients in May. In conducted study in U.S.A by Diane K, et al. [17] the Metformin 
had captured percent of 33 of prescriptions. In India by Vengurlekar, et al. [18] Metformin percent was 27\%.

Utilization of antidi abetic was high in female more than male in April while in May it was high in male more than female. For the DID metformin was increased in May in compared to April, the same result was occurred also for the zother oral antidiabetics including glibencalamide, glimperide and pioglitazone. Average DDD in was more in May than April. Different findings also reported in a study conducted by Benitez J, et al. in Spain. That means all of oral antidiabetic utilization in Elbanjadeed Hospital was under use in comparing with this study. In another study conducted by Mousnad, et al. [13] in Malaysia showed that DDD and DID for all antidiabetic was low, Comparing to this study also utilization of antidiabetic in Elbanjadeed Hospital was under use. Not follow of standard guidelines and poor of rational prescribing might be main reasons for these differences. There was a highly significant difference between DDDs values and also highly significant difference between DID values this was considered as a problem because significant differences usually occur between different years and in this study occur between two months that means updating of treatment guidelines and drug prescribing patter was very poor in this health care system.

Average number of drug per prescription was generally high. Percentage of generic drug prescribed and no. of drugs in EML were more than half. Average drug cost per prescription was (115.8 SD about $16.54 \$$ ). In a study conducted by En were, et al. [15] in Nigeria showed that the average number of drugs prescribed at the diabetic clinic was high. Percentage of drugs prescribed in their generic name was less than our study which was less than half. Percentage of drugs prescribed from the hospital essential drug list (EDL) was very high. The average cost of medications to the patients were $(1.31 \$)$. In another study Number of drugs prescribed by generic name was less than half, Number of drugs prescribed from essential drug list was more than half. Average cost of prescription was high in Elbanjadeed Hospital because the average number of drugs per prescription was high and there is a general increase in cost of drugs in Sudan generally utilization of oral antidiabetics were under use for all oral antidiabetics that were included in our study.

\section{Conclusions and Recommendations}

\section{Conclusions}

The study showed that male was predominant more than female. DM prevalence was more towards the active age group (41-60) which really public problem. Alimentary tract and metabolism was most prescribed drug class and Metformin was the most prescribed antidiabetic drug.
Cardiovascular problems which affected (27\%) of the diabetic patients was the most disorders associated with DM. Average number of drug per prescription and average cost per prescription determined by this study were high. Due to currency inflation during last years in Sudan, cost of drugs generally is high. There was no statistically significant difference between the two months period of the study. The results showed a very weak positive correlation between drug utilization the age; and weak positive correlation with gender.

\section{Recommendations}

Elbanjadeed Hospital now has a classified medicine list for oral antidiabetic drugs according to an international standard classification system (ATC classification system). Prior to this study, no such list was available.

\section{This Study Recommends the following}

- Prescribers in Elbanjadeed Hospital should be followed the standard treatment guidelines and rational prescribing.

- Statistic on medicine should be used in the hospital for preparing drug utilization reports and to provide information for decisions makers and health care providers.

- Further researches are needed for in-depth analysis of the drug utilization trends in Elbanjadeed Hospital.

- Full drug utilization review needed to explore the reasons behind the under use of oral antidiabtic dugs in Elbanjadeed Hospital.

\section{References}

1. Barbara G Wells, Joseph T, Terry L, Schwinghammer, Cecily V (2009) Pharmacotherapy. $7^{\text {th }}$ (Edn.), chapter 4, pp: 210.

2. Russell JG, Harris ND (2008) Pathology and therapeutic for pharmacists. $3^{\text {rd }}$ (Edn.), Chapter 8, pp: 582.

3. Steyn R, Burger JR, Serfontein JHP, Lubbe MS (2007) Investigation Into The prescribing Patterns And Cost Of Antidiabetic Medicine In South Africa. Health SA Gesondheid 12(3): 26-36.

4. Saeedi P, Petersohn I, Salpea P, Malanda B, Karuranga $S$, et al. (2019) Global and regional diabetes prevalence estimates for 2019 and projections for 2030 and 2045: Results from the International Diabetes Federation Diabetes Atlas, 9th edition. Diabetes Research and Clinical Practice. 157: 107843.

5. Ahmed AM, Ahmed NH (2001) Diabetes mellitus in Sudan: the size of the problem and the possibilities of 
efficient care. Practical Diabetes International 18(9): 324-327.

6. (2011) World Health Organization-NCD Country Profiles.

7. Evaluation of Prescription Patterns and Cost of Illness of Type-2 Diabetic Patients in a Tertiary Health Care Hospital.

8. WHO (2013) Guidelines for ATC classification and DDD assignment.

9. Mabadeje BB, Massele A, laing RO, Ofori D, Ross D, et al. (1993) How to investigate drug use in health facilities selected drug use indicators WHO.

10. Upadhyay DK, Palaian S, Ravi Shankar P, Mishra P, Sah AK (1998) Prescribing pattern in diabetic outpatients in a tertiary care teaching hospital in Nepal 2007.

11. Rathmann W1, Haastert B, Roseman JM, Gries FA, Giani G (1998) Prescription drug use and costs among diabetic patients in primary health care practices in Germany. Diabetes Care 21(3):389-397.

12. Kennedy, Dianne L, Piper, Joyce M, Baum, et al. (1988) Trends in Use of Oral Hypoglycemic Agents 1964-1986.
Diabetes Care 11(7): 558-562.

13. Mousnad M, Ibrahim M (2009) PDB8 Antidiabetic Drug Utilization in a University Health Care Setting. Value in Health 12(7).

14. Leelavathi D Acharya (2012) India Evaluation of Prescription Patterns and Cost of Illness of Type-2 Diabetic Patients in a Tertiary Health Care Hospital.

15. Enwere OO, Salako BL, Falade CO (2006) Prescription and Cost Consideration at a Diabetic Clinic in Ibadan, Nigeria. Annals of Ibadan Postgraduate Medicine 4(2): 35-39.

16. Jenny L, Arifull JM, Haran JS, Myalll JM, Das R, et al. (2012) Age and Gender-Based Utilization Pattern of Antidiabetic Drugs In Ajman, UAE.

17. Wysowski DK, Armstrong G, Governale L (2003) Rapid Increase in the Use of Oral Antidiabetic Drugs in the United States, 1990-2001. Diabetes Care 26(6): 18521855.

18. Vengurlekar S, Shukla P, Patidar P, Bafna R, Jain S (2008) Prescribing pattern of antidiabetic drugs in Indore city hospital. Indian J Pharm Sci 70(5): 637-640. 